\title{
Management of gastric cancer with liver metastasis in a pregnant woman
}

\author{
Numan Cim ${ }^{1, *}$, Erbil Karaman ${ }^{1}$, Osman Toktas ${ }^{2}$, Gulhan Gunes ${ }^{1}$, Erkan Elci ${ }^{1}$, Esra Andıc ${ }^{1}$, Serhat \\ Ege $^{3}$, Recep Yildizhan ${ }^{1}$ \\ ${ }^{1} Y$ uzuncu Yil University Faculty of Medicine, Department of Obstetric and Gynecology, Van, Turkey \\ ${ }^{2}$ Y uzuncu Yil University Faculty of Medicine, Department of General Surgery, V an, Turkey \\ ${ }^{3}$ Republic of Turkey-Ministry of Health, Diyarbaker Gazi Yasargil Training and Research Hospital, Clinic of \\ Obstetrics and Gynecology, Turkey
}

\begin{abstract}
The complaints and symptoms of gastric cancer are non-specific and many of these symptoms are frequently masked by factors related to normal pregnancy. A 38 year-old woman with G10P8A1 and at 31 weeks of pregnancy was admitted to a maternity outpatients' clinic complaining of nausea, vomiting and constipation during the previous 4 days. On palpation a solid lesion of $1.5 \mathrm{~cm}$ was palpable in the subxiphoid region. A solid nodule with largest diameter of $5 \mathrm{~cm}$ was observed on the liver and suspected to indicate metastasis. Under endoscopic examination, ulcerovegetan, fragile and malignant mass that surrounded and narrowed the gastric antrum and extended to an angular notch was seen. The biopsy that was taken from lesion was revealed to be the result of a malignant epithelial tumor of poorly differentiated adenocarcinoma. During the explorative laparotomy, a tumoral mass with multiple peripheral lymphadenopathies was observed in the posterior gastric antrum, pancreas and colon median entry-invasive artery. Multiple metastases were present in the omentum and liver with a large amount of acid in the abdomen. The patient was considered inoperable due to the gastric cancer being in a terminal stage and adjuvant chemotherapy was planned.

Early diagnosis and treatment is important for the prognosis of gastric cancer. The complaints and symptoms of gastric cancer are non-specific and many of these symptoms are similar to those seen during a normal pregnancy. If the same symptoms recur during pregnancy or there is a failure to respond to therapy, the gastrointestinal system should be evaluated endoscopically.
\end{abstract}

Key Words: Pregnancy, liver metastasis, gastric cancer

\section{Introduction}

Gastric cancer arises from the upper gastrointestinal tract and is usually seen before the age of 40 . The incidence of gastric cancer varies across the world. Most of the studies and case reports are from Japan which has the highest incidence of gastric cancers worldwide and its prevalence in Japan is 80 per 100.000. The prevalence in Western Europe and the US is 10-40 per 100.000 (1). Each year approximately 700.000 people die worldwide due to gastric cancer (2). The incidence of cancer during pregnancy is rare at approximately $0.1 \%$, and that of gastric cancer is 0.026 to $0.1 \%$ of all pregnancies (3).

Nonspecific complaints related to the gastrointestinal system are seen during the early stage of gastric cancer cases. In the advanced stages, complaints such as nausea-vomiting, abdominal pain, postprandial saturation, loss of appetite, dysphagia and weight loss may be seen (4).

We aim to present the case of a pregnant woman with gastric cancer with liver metastasis and to emphasize the importance of early diagnosis and treatment.

\section{Case report}

A 38 year-old woman with G10P8A1 was admitted to a maternity outpatient clinic complaining of nausea, vomiting and constipation during the previous 4 days. These complaints were occasional and infrequent. She stated that antiemetic and anti-reflux medications had been prescribed for earlier complaints, and that when she used these drugs there was a decrease in her symptoms. She had lost about $24 \mathrm{~kg}$ weight in the previous month. No remarkable findings were noted in her history. In abdominal examination, 
the abdomen was comfortable, and there was no defense or rebound. On palpation a solid lesion of $1.5 \mathrm{~cm}$ was palpable in the subxiphoid region. On obstetric examination, the ultrasound revealed a singleton intrauterine fetus with growth corresponding to 31 weeks of pregnancy consistent with last menstrual period. No obstetric pathologies were noted on either the placenta or fetus. Abdominal examination could not be performed due to the gravid uterus, however, a solid nodule with a largest diameter of $5 \mathrm{~cm}$ was observed on the liver and suspected to indicate metastasis. On endoscopic examination, an ulcerovegetan, fragile and malignant mass that surrounded and narrowed the gastric antrum and extended to an angular notch was seen and which would not allow endoscope to pass beyond it. The biopsy that was taken from lesion revealed a malignant epithelial tumor of poorly differentiated adenocarcinoma. For fetal lung maturity we applied $6 \mathrm{mg}$ of betamethasone intramuscularly every 24 hours to the pregnant woman. Delivery was induced vaginally and the patient gave birth to a $1440 \mathrm{gr}$ male baby with Apgar scores 6-8. The patient was transferred to the general surgery clinic and she was operated on for stomach cancer on the $7^{\text {th }}$ postpartum day. On the explorative laparotomy, a tumoral mass with multiple peripheral lymphadenopathies was observed in the posterior gastric antrum, pancreas and colon median entry-invasive artery. Multiple metastases were present in the omentum and liver with large amount of acid in the abdomen were present. The patient was judged inoperable due to the terminal stage of the gastric cancer and adjuvant chemotherapy was planned. The patient underwent omentectomy and palliative gastrojejunostomy with multiple biopsies taken from the liver and lymphadenopathies. During the postoperative follow-up, pancytopenia and electrolyte imbalance developed. Replacement therapies were applied. On the 5 th post-operative day, cardiopulmonary resuscitation was applied due to cardiac arrest. However, the patient did not respond to the intervention and died.

\section{Discussion}

Because of physiological changes during pregnancy and the pressure of the gravid uterus on abdominal organs, such as the stomach and intestines, as well as hormones produced during pregnancy hormones, nausea, vomiting, nonspecific abdominal pain and symptoms of constipation may be seen. In our case, a woman who was 31 weeks pregnant was admitted to our clinic with nonspecific complaints such as nausea and vomiting which responded to anti-emetic drugs.

In general, pregnant women do not pay attention to these complaints and the symptoms may also be attributed to emesis or hyperemesis gravidarum in the early weeks of pregnancy. Hyperemesis gravidarum usually continues until the 20th week of pregnancy. If it continues in the second trimester and does not respond to treatment for nausea-vomiting or gastrointestinal complaints, diseases of the gastrointestinal tract should also be considered in a differential diagnosis (5). In our case, the symptoms continued until the third trimester and a palpable mass with a size of $1.5 \mathrm{~cm}$ in the subxiphoid region led us to suspect a malignancy; we therefore performed a gastric endoscopy. The biopsy was taken from the region of the existing lesion. The pathological examination of the biopsy showed a malignant epithelial tumor compatible with poorly differentiated adenocarcinoma. In the literature, the histologic subtype of adenocarcinoma of gastric cancer has been identified more than other types in both men and women $(6,7)$.

The lesions in our case started from the gastric antrum, extended to an angular notch and narrowed the gastric antrum round. The localization of the tumor is important in the treatment and prognosis of gastric cancer. While the prognosis for tumors arising from the proximal stomach is bad, the prognosis for tumors arising in the middle third of the stomach may be better after the surgery (8).

In gastric cancer, the other situations associated with a bad prognosis are vascular invasion, regional lymphadenopathy and liver metastases (9). In the abdominal ultrasonography of our case the solid nodule had a diameter of $5 \mathrm{~cm}$ and largest vascularity was observed in liver, and these findings were thought to indicate metastasis as described in the literature. In cases of suspected or diagnosed gastric cancer, liver metastases should be considered if a high serum alphafetoprotein (AFP) level has been identified (9). This high level is important in the cases of those who are not pregnant, but in high serum AFP levels increase physiologically during the pregnancy. So this situation can cause confusion.

In gastric cancer cases, surgical treatment can be applied during pregnancy. In cases where it is necessary, chemotherapy or radiotherapy should be postponed to the period after pregnancy (10). However, Nishie et al. applied chemotherapy to a pregnant woman with metastatic gastric adenocarcinoma who was 23 weeks pregnant and 
delivered her at 32 weeks with a caesarean section. The child continued to show no abnormalities at 12 months after delivery (11). Ueno et al. reported that the treatment of gastric cancer during pregnancy depends on the stage of the disease, the week of pregnancy and the development of fetus at the time of diagnosis. They stated that when gastric cancer is diagnosed prior to 22 weeks gestation, the mother should be treated after the termination of the pregnancy by abortion. After 28 weeks gestation, treatment should be administered after early delivery, as was seen in our case (3). Surgical treatment was planned for our case after delivery at 32 weeks gestation. During the operation, the case was judged inoperative as the gastric cancer was in the terminal stage and it was decided to apply chemotherapy.

The 5-year survival rate for gastric cancer is $25 \%$ in Europe. The prognosis in gastric cancer with liver metastases is even worse: The 5-year survey does not exceed $10 \%$. The average survival of those who are not treated is around 3-5 months $(12,13)$. Treatment of cases of gastric cancer with liver metastases is controversial. Surgical treatment is appropriate when there is only one lesion in the liver and it is smaller than $5 \mathrm{~cm}$. But such cases are not common. Scientific evidence does not exist for the other therapies such as palliative gastrectomy and radiofrequency ablation of hepatic arterial infusion. Systemic chemotherapy promises longer survival and good quality of life in these situations (14).

The diagnosis of gastric cancer is often delayed during pregnancy. In their review, Jaspers et al. reported on 92 gastric cancer cases and stated that almost all were at the advanced stage (15). The management of gastric cancer during pregnancy is dependent on the stage of the tumor and the gestational age and immaturity of the fetus. A study by Sakamoto et al. analysed a total of 137 cases of gastric cancer and found that $92.5 \%$ of patients had advanced gastric cancer at the time of diagnosis and only $45.3 \%$ of cases underwent gastrectomy, including incomplete resection, and that the prognosis was extremely poor with a 1 year survival rate of $14.5 \%$ (16).

The management of gastric cancer during pregnancy is clear and well-established when the gestational age is below 22 weeks or above 28 weeks. However, it is difficult to decide whether to continue or terminate pregnancy when a pregnant woman is diagnosed with gastric cancer at 22-28 weeks pregnancy (17). There is no consensus and a lack of data regarding gastric cancer management during this period of time. We think that the treatment strategy should be individualized with regard to the stage of disease whether metastatic or not and the availability of an advanced neonatal intensive care unit. So the patient should be evaluated and followed by a team including both a perinatal obstetrician and oncologist.

In the abdominal exploration of our case, tumor formations were observed in the posterior gastric antrum, pancreas and colon median entry-invasive artery, as were multiple environmental lymphadenopathies and common acid in the abdomen and multiple metastases in the omentum and liver, and these findings agreed with the finding of advanced gastric cancer. As noted in the literature, the survival rate is short in advanced gastric cancer (3).

Consequently, the case of a pregnant woman with advanced-stage gastric cancer has been presented here. It is well known that the symptoms of gastric cancer are non-specific. Due to many of these complaints also being present during pregnancy, a diagnosis may be difficult. In situations continuing for a long time and in the presence of gastrointestinal symptoms that fail to respond to treatment, digestive system-derived pathologies should be considered and examinations for these should be carried out. The prognosis for gastric cancer during pregnancy can be improved by early diagnosis and physician should be alerted when non-specific gastrointestinal complaints are not resolved and continue into the second and third trimesters of the pregnancy.

Conflict of interest: The authors declare that they have no conflict of interest.

Informed consent: Written informed consent was obtained from a first-degree relative of the patient with regard to the data and accompanying images.

\section{References}

1. Parkin DM, Bray F, Ferlay J, Pisani P. Estimating the world cancer burden: Globocan 2000. Int J Cancer 2001; 94: 153156.

2. Parkin DM, Baray F, Ferlay J, Pisani P. Global Cancer Statistics, 2002. Ca Cancer J Clin 2005; 55: 74-108.

3. Ueo H, Matsuoka H, Tamura S, Sato K, Tsunematsu Y, Kato T. Prognosis in gastric cancer associated with pregnancy. World J Surg 1991; 15: 293-298.

4. Kaurah P, Huntsman DG. Hereditary Diffuse Gastric Cancer. In: Pagon RA, Adam MP, Ardinger HH, Wallace SE, Amemiya A, 
Cim et al / Gastric cancer in pregnant woman

Bean LJH, Bird TD, Dolan CR, Fong CT, Smith RJH, Stephens K, editors. GeneReviews $^{\circledR} \quad$ [Internet]. Seattle (WA): University of Washington, Seattle; 1993-2015. 2014.

5. Yoshida M, Matsuda H, Furuya K. Successful treatment of gastric cancer in pregnancy. Taiwan J Obstet Gynecol 2009; 48: 282-285.

6. Almasi Z, Rafiemanesh $H$, Salehiniya $H$. Epidemiology characteristics and trends of incidence and morphology of stomach cancer in Iran. Asian Pac J Cancer Prev 2015; 16: 2757-2761.

7. Hajmanoochehri F, Mohammadi N, Nasirian N, Hosseinkhani M. Patho-epidemiological features of esophageal and gastric cancers in an endemic region: a 20-year retrospective study. Asian Pac J Cancer Prev 2013; 14: 3491-3497.

8. Haidari M, Nikbakht MR, Pasdar Y, Najaf F. Trend analysis of gastric cancer incidence in Iran and its six geographical areas during 2000-2005. Asian Pac J Cancer Prev 2012; 13 : 3335-3341.

9. Cardinale V, De Filippis G, Corsi A, et al. An isolate alpha-fetoprotein producing gastric cancer liver metastasis emerged in a patient previously affected by radiation induced liver disease. World J Hepatol 2013; 5: 398-403.

10. Al- İbrahim A. Parrish J, Dunn E, Swallow C, Maxwell C. Pregnancy and maternal outcomes in women with prior or current gastrointestinal malignancies. J Obstet Gynaecol Can 2014; 36: 34-41.

11. Nishie H, Mizushima T, Suzuki Y, et al. Chemotherapy treatment of a pregnant woman with progressive gastric cancer. Intern Med 2015; 54: 1207-1212.

12. Verdecchia A, Francisci S, Brenner H, et al EUROCARE-4 Working Group. Lancet Oncol 2007; 8: 784-796.

13. Howlader N, Noone AM, Krapcho M, Garshell J, Miller D, Altekruse SF, Kosary CL, Yu M, Ruhl J, Tatalovich Z,Mariotto A, Lewis DR, Chen HS, Feuer EJ, Cronin KA (eds). SEER Cancer Statistics Review, 19752011, National Cancer Institute. Bethesda, $\mathrm{MD}$, http://seer.cancer.gov/csr/1975_2011/, based on November 2013 SEER data submission, posted to the SEER web site, April 2014.

14. Jerraya H, Saidani A, Khalfallah M, Bouasker I, Nouira R, Dziri C. Management of liver metastases from gastric carcinoma: where is the evidence? Tunis Med 2013; 91: 1-5.

15. Jaspers VKI, Gillessen A, Quakernack K. Gastric cancer in pregnancy: Do pregnancy, age or female sex alter the prognosis? Case reports and review. Eur J Obstet Gynecol Reprod Biol 1999; 87: 13-22.

16. Sakamoto K, Kanda T, Ohashi M, et al. Management of patients with pregnancyassociated gastric cancer in Japan: a minireview. Int J Clin Oncol 2009; 14: 392-396.

17. Ueo H, Okudaira Y, Hirabayashi M. Gastric cancer associated with pregnancy. A case report of early gastric cancer and review of 100 cases in Japan (in Japanese). J Jpn Soc Clin Surg 1989; 50: 312-318. 